\title{
New Bifurcation Critical Criterion of Flip-Neimark-Sacker Bifurcations for Two-Parameterized Family of $n$-Dimensional Discrete Systems
}

\author{
Shengji Yao \\ Department of Mechanical Engineering, McMaster University, 1280 Main Street West, \\ JHE-308/A, Hamilton, ON, Canada L8S 4L7 \\ Correspondence should be addressed to Shengji Yao, yao@mcmaster.ca
}

Received 18 April 2012; Accepted 21 July 2012

Academic Editor: Francisco Solis

Copyright (C) 2012 Shengji Yao. This is an open access article distributed under the Creative Commons Attribution License, which permits unrestricted use, distribution, and reproduction in any medium, provided the original work is properly cited.

\begin{abstract}
A new bifurcation critical criterion of flip-Neimark-Sacker bifurcation is proposed for detecting or anticontrolling this type of codimension-two bifurcation of discrete systems in a general sense. The criterion is built on the properties of coefficients of characteristic equations instead of the properties of eigenvalues of Jacobian matrix of nonlinear system, which is formulated using a set of simple equalities and inequalities consisting of the coefficients of characteristic polynomial equation. The inequality conditions enable us to easily pick off the fake parameter domain whereas the equality conditions are used to accurately locate the critical bifurcation point. In particular, after the bifurcation parameter piont is determined, the inequality conditions can be used to figure out the feasible region of other system parameters. Thus, the criterion is suitable for two-parameterized family of $n$-dimensional discrete systems. As compared with the classical critical criterion (or definition) of flip-Neimark-Sacker bifurcation stated in terms of the properties of eigenvalues, the proposed criterion is preferable in anticontrolling or detecting the existence of flip-Neimark-Sacker bifurcation in high-dimension nonlinear systems, due to its explicit parameter mechanism of the bifurcation.
\end{abstract}

\section{Introduction}

With the development of bifurcation analysis [1-3] and nonlinear control theories [4, 5], people become interested in active utilization of the bifurcation characteristics. The seminal work of Chen and his coauthors [6, 7] presented the concept of anticontrol of bifurcation. Similar to anticontrol of chaos, anticontrol of bifurcation is aimed at actively creating a certain bifurcation with desired dynamic properties at a prespecified system parameter point via 
control method in order to utilize bifurcation characteristics. Notice that among the classic bifurcation analysis, one can determine the type of bifurcation and its existence based on the critical criterion or definition of bifurcation. Some developed software packages such as MATCONT [8] that allow one to numerically search for the curves of equilibria, limit points, Hopf points, limit cycles, period-doubling bifurcation points of limit cycles, and fold bifurcation points of limit cycles by using a prediction-correction continuation algorithm. However, Wen et al. have pointed out the fact $[9,10]$ that for anticontrol of bifurcation in high-dimension nonlinear systems, the classical critical criteria or definitions of bifurcations described by the properties of eigenvalue of Jacobian matrix are not suitable for the design of gains, especially for complicated codimenison bifurcations with multiparameters and various bifurcating solutions. It is significant for anticontrol of bifurcation to develop explicit critical criterion of the corresponding bifurcation. Bifurcation, a qualitative change of dynamical properties, is a critical phenomena related to the loss of system stability. Thus, it is possible to establish new explicit critical criteria based on the existing stability criteria without using eigenvalues, which give the stability conditions with significant computational simplification for multiparameter systems, such as the Schur-Cohn stability criterion [11, 12] and the formulation of stability limit in terms of the Jacobian matrix and its bialternate product [13]. Recently, Wen and the coauthors [14-19] have systematically developed some critical criteria of Hopf bifurcation at nonresonance or resonance, period-doubling bifurcation, degenerate Hopf bifurcation, and interaction of Hopf-Hopf bifurcation in high-dimensional systems. The advantages of the criteria independent of eigenvalue computations are obvious in the applications to anticontrol of bifurcations or detecting the existence $[20,21]$ as compared with the methodologies of numerical search for bifurcation points [8] or the classical bifurcation definitions [1-3].

Flip-Neimark-Sacker bifurcation is one of so-called codimension two singularities of discrete time systems. The bifurcation may give rise to rich bifurcation outcomes depending on two-parameter unfolding at the bifurcation point, including unstable (or stable) fixed point, stable (or unstable) limit circle, stable (or unstable) fixed points of order two, and a couple of stable (or unstable) invariant circles [22, 23]. It should be stressed that this bifurcation occurs only in high-dimensional $(\geq 3)$ systems, and its bifurcation point involves with at least two bifurcation parameters. Subject to the classical critical criterion stated in terms of the properties of eigenvalues $[2,22]$, the main idea of detecting the existence of the bifurcation in the literature is to directly compute and check all eigenvalues of the Jacobian matrix at each parameter point in the parameter plane. Since the difficulty in the eigenvalue computation point by point in the parameter plane, it is in general an arduous task to detect a bifurcation point in practical physical systems. The same difficulty exists in the design of gains for anticontrol of this complicated codimension bifurcation. Wen and $\mathrm{Xu}[18,24]$ discussed a critical criterion without using eigenvalues and its application to anticontrol of this bifurcation. However, their critical criterion is only limited to the four-dimensional dynamical systems and not universal in a general sense.

The main purpose of this paper is to study a criterion without using eigenvalues for anticontrolling or detecting the flip-Neimark-Sacker bifurcation in discrete time systems in a general sense. With the application of the criterion to two-parameterized family of $n$ dimensional discrete systems, the effects of the parameters on the bifurcation may be explicitly formulated. Numerical examples show that the proposed criterion is more convenient and efficient to analyze this kind of complicated bifurcation than the classical bifurcation critical criterion. 


\section{Problem Formulation and Preliminaries}

Consider an $n$-dimension nonlinear discrete time system (or map) in the general form,

$$
x_{k+1}=f_{\mu}\left(x_{k}\right),
$$

where $x_{k+1}, x_{k} \in R^{n}$ are the state vectors, $k$ is the iterative index, and $f_{\mu}$ denotes the nonlinear function vector. $\mu \in R^{m}$ is the system parameter vector. For flip-Neimark-Sacker bifurcation (also called as Hopf-flip interaction bifurcation), we have $\mu=\left(\mu_{1}, \mu_{2}\right)^{T} \in R^{2}$ for this type of codimension-two bifurcation if $\mu$ is limited to the bifurcation parameters. It is often convenient to state the definition of flip-Neimark-Sacker bifurcation for map (2.1) as the following Definition 2.1.

Definition 2.1 (see $[2,22,23]$ ). Suppose that $f_{\mu}$ has a fixed point $x_{0}$. A flip-Neimark-Sacker bifurcation occurs at a bifurcation point $\mu=\mu_{0}$ if and only if system (2.1) satisfies the following conditions:

(C1) eigenvalue assignment: the Jacobian matrix $D_{x_{k}} f_{\mu}\left(x_{0}\right)$ has a pair of complex conjugate eigenvalues $\lambda_{1}(\mu)$ and $\bar{\lambda}_{1}(\mu)$ with $\left|\lambda_{1}\left(\mu_{0}\right)\right|=1$, one real eigenvalue $\lambda_{3}\left(\mu_{0}\right)=-1$, and the rest $\lambda_{j}(\mu)$ with $\left|\lambda_{j}\left(\mu_{0}\right)\right|<1, j=4, \ldots, n$;

(C2) transversality condition: $\quad \partial\left|\lambda_{1}(\mu)\right| /\left.\partial \mu_{1}\right|_{\mu=\mu_{0}} \neq 0, \quad \partial\left|\lambda_{1}(\mu)\right| /\left.\partial \mu_{2}\right|_{\mu=\mu_{0}} \neq 0$, $\partial\left|\lambda_{3}(\mu)\right| /\left.\partial \mu_{1}\right|_{\mu=\mu_{0}} \neq 0$, and $\partial\left|\lambda_{3}(\mu)\right| /\left.\partial \mu_{2}\right|_{\mu=\mu_{0}} \neq 0$, where $\mu_{1}, \mu_{2}$ are the bifurcation parameters;

(C3) nonresonance condition: $\lambda_{1}^{m}\left(\mu_{0}\right) \neq 1$ where $m=3,4,5, \ldots$

Definition 2.1 gives the classical critical criterion of flip-Neimark-Sacker bifurcation which is used to determine the existence of the bifurcation as usual. The type and stability of bifurcation solutions depend on the nonlinear property of map $f_{\mu}$ and the parameter unfolding of $\mu_{1}$ and $\mu_{2}$ at $\mu=\mu_{0}$ [22, 23]. As shown in Definition 2.1, the classical critical criterion is stated in terms of the properties of eigenvalues. Furthermore, it follows from the condition (C1) that the bifurcation occurs only in three or higher dimensional systems. It is well known that it is very difficult or even infeasible to obtain the analytical expressions of all eigenvalues with respect to $\mu$ in high-dimensional systems. This implies that the classical critical criterion fails to explicitly formulate the effect of parameters on the bifurcation. In order to detect the existence of this type of codimension-two bifurcation, we have to resort to eigenvalue computation point by point by scanning the parameter space $\left(\mu_{1}, \mu_{2}\right)$ as shooting an arrow without a target. In particular, it is a nontrivial task to check the transversality condition without the analytical expressions of the derivatives of eigenvalue modulus. This implies that it is in general an arduous task to detect a bifurcation point $\mu_{0}=\left(\mu_{10}, \mu_{20}\right)^{T}$ in practical physical systems by applying the classical critical criterion.

In the next section, we will explore a new critical criterion of flip-Neimark-Sacker bifurcation in a general sense. The criterion is formulated using a set of equalities or inequalities consisting of the coefficients of characteristic polynomial equation, which is independent of the computation of eigenvalues. This property may overcome the aforementioned difficulties for the classical critical criterion. 


\section{Critical Criterion without Using Eigenvalues}

In order to obtain the criterion of flip-Neimark-Sacker bifurcation without using eigenvalues, we write the characteristic polynomial of map (2.1) at the fixed point $x_{0}$ as

$$
p_{\mu}(\lambda)=a_{0} \lambda^{n}+a_{1} \lambda^{n-1} \cdots+a_{n-1} \lambda+a_{n}
$$

where $a_{0}=1$ and $a_{j}=a_{j}(\mu), j=1, \ldots, n$. Define the sequence of determinants [11], $\Delta_{k}^{ \pm}(\mu)=1$ for $k \leq 0$, and $\Delta_{1}^{ \pm}(\mu), \ldots, \Delta_{n}^{ \pm}(\mu)$, where

$$
\Delta_{j}^{ \pm}(\mu)=\left|\left(\begin{array}{ccccc}
1 & a_{1} & a_{2} & \cdots & a_{j-1} \\
0 & 1 & a_{1} & \cdots & a_{j-2} \\
0 & 0 & 1 & \cdots & a_{j-3} \\
\cdots & \cdots & \cdots & \cdots & \cdots \\
0 & 0 & 0 & \cdots & 1
\end{array}\right) \pm\left(\begin{array}{ccccc}
a_{n-j+1} & a_{n-j+2} & \cdots & a_{n-1} & a_{n} \\
a_{n-j+2} & a_{n-j+3} & \cdots & a_{n} & 0 \\
\cdots & \cdots & \cdots & \cdots & \cdots \\
a_{n-1} & a_{n} & \cdots & 0 & 0 \\
a_{n} & 0 & \cdots & 0 & 0
\end{array}\right)\right|, \quad j=1, \ldots, n .
$$

Furthermore, the following Lemma 3.1 describing the critical criterion of Hopf bifurcation is needed to serve the purpose of formulating a new criterion of flip-Neimark-Sacker bifurcation.

Lemma 3.1 (see [9]). For map (2.1), the following conditions (H1)-(H3):

(H1) eigenvalue assignment: $\Delta_{n-1}^{-}\left(\mu_{0}\right)=0, p_{\mu_{0}}(1)>0,(-1)^{n} p_{\mu_{0}}(-1)>0, \Delta_{n-1}^{+}\left(\mu_{0}\right)>0$, $\Delta_{j}^{ \pm}\left(\mu_{0}\right)>0, j=n-3, n-5, \ldots, 1$ (or 2 ), when $n$ is even (or odd, resp.);

(H2) transversality condition: $\partial \Delta_{n-1}^{-}(\mu) /\left.\partial \mu_{j}\right|_{\mu=\mu_{0}} \neq 0, j=1,2$, and $\mu_{1}, \mu_{2}$ are the bifurcation parameters;

(H3) nonresonance condition: $\cos (2 \pi / m) \neq \psi$ where $m=3,4,5, \ldots$ and $\psi=1-$ $0.5 p_{\mu_{0}}(1) \Delta_{n-3}^{-}\left(\mu_{0}\right) / \Delta_{n-2}^{+}\left(\mu_{0}\right)$, are equivalent to the following conditions (D1)-(D3) from the classical critical criterion of Hopf bifurcation for maps:

(D1) eigenvalue assignment: the Jacobian matrix $D_{x_{k}} f_{\mu}\left(x_{0}\right)$ has a pair of complex conjugate eigenvalues $\lambda_{1}(\mu)$ and $\bar{\lambda}_{1}(\mu)$ with $\left|\lambda_{1}\left(\mu_{0}\right)\right|=1$, and the rest $\lambda_{j}(\mu)$ with $\left|\lambda_{j}\left(\mu_{0}\right)\right|<1$, $j=3, \ldots, n$;

(D2) transversality condition: $\partial\left|\lambda_{1}(\mu)\right| /\left.\partial \mu_{j}\right|_{\mu=\mu_{0}} \neq 0, j=1,2$;

(D3) nonresonance condition: $\lambda_{1}^{m}\left(\mu_{0}\right) \neq 1$ where $m=3,4,5, \ldots$.

Based on Lemma 3.1, the following conditions (T1)-(T3) in Theorem 3.2 can be formulated to establish a new critical criterion of flip-Neimark-Sacker bifurcations in a general sense.

Theorem 3.2. For a general n-dimensional map (2.1), the flip-Neimark-Sacker bifurcation occurs at $\mu_{0}$ if and only if the following conditions (T1)-(T3) hold:

(T1) eigenvalue assignment: $p_{\mu_{0}}(-1)=0, \Delta_{n-2}^{-}\left(\mu_{0}\right)=0, p_{\mu_{0}}(1)>0, \Delta_{n-2}^{+}\left(\mu_{0}\right)>0, \Delta_{j}^{ \pm}\left(\mu_{0}\right)>0$, $j=n-4, n-6, \ldots, 1$ (or 2 ), when $n$ is odd (or even, resp.), and

$$
(-1)^{n-1} \sum_{k=1}^{n}\left((-1)^{n-k} \sum_{i=1}^{k}\left((-1)^{k-i} a_{i-1}\right)\right)>0,
$$


(T2) transversality condition:

$$
\begin{gathered}
\left.\frac{\partial \Delta_{n-2}^{-}(\mu)}{\partial \mu_{j}}\right|_{\mu=\mu_{0}} \neq 0, \quad j=1,2, \\
\frac{\sum_{i=1}^{n} a_{i j}^{\prime}(-1)^{n-i}}{\sum_{k=1}^{n}(n-k+1)(-1)^{n-k} a_{k-1}} \neq 0, \quad j=1,2,
\end{gathered}
$$

where $a_{i j}^{\prime}$ stands for the derivative of $a_{i}(\mu)$ with respect to $\mu_{j}$ at $\mu=\mu_{0}$, and $\mu_{1}, \mu_{2}$ are the bifurcation parameters,

(T3) nonresonance condition: $\cos (2 \pi / m) \neq \psi$ where $m=3,4,5, \ldots$ and $\psi=1$ $\left(p_{\mu_{0}}(1) \Delta_{n-4}^{-}\left(\mu_{0}\right) / 4 \Delta_{n-3}^{+}\left(\mu_{0}\right)\right)$, where we have $\Delta_{k}^{ \pm}(\mu)=1$ if $k \leq 0$.

Proof. If the conditions (T1)-(T3) in Theorem 3.2 are equivalent to the conditions (C1)-(C3) in Definition 2.1, then Theorem 3.2 stands for a critical criterion of flip-Neimark-Sacker bifurcations for maps. First of all, it should be noticed that the equality $p_{\mu_{0}}(-1)=0$ in the condition (T1) implies that the Jacobian matrix $D_{x_{k}} f_{\mu}\left(x_{0}\right)$ has one real eigenvalue equal to -1 at $\mu=\mu_{0}$, and vice versa. In other words, at $\mu=\mu_{0}$, we may rewrite the characteristic polynomial $p_{\mu}(\lambda)$ in (3.1) as

$$
p_{\mu_{0}}(\lambda)=(\lambda+1) \tilde{p}_{\mu_{0}}(\lambda)
$$

where $\tilde{p}_{\mu_{0}}(\lambda)=\lambda^{n-1}+b_{1} \lambda^{n-2} \cdots+b_{n-3} \lambda+b_{n-1}$. All what we have to do further is employ Lemma 3.1 to show that $\tilde{p}_{\mu_{0}}(\lambda)=0$ satisfies the conditions $(\mathrm{H} 1)-(\mathrm{H} 3)$ subject to the conditions (T1)-(T3).

It follows from (3.5) that $p_{\mu_{0}}(1)=2 \tilde{p}_{\mu_{0}}(1)$. Thus, $p_{\mu_{0}}(1)>0$ implies $\tilde{p}_{\mu_{0}}(1)>0$. By expanding the right-hand side of (3.5), one can easily obtain the relationship of the coefficients $a_{m}$ in (3.1) and $b_{m}$ in (3.5) as follows:

$$
a_{m}=b_{m}+b_{m-1}
$$

where $m=1, \ldots, n, b_{0}=1$ and $b_{j}=0$ if $j>(n-1)$ or $j<0$. Substituting (3.6) into (3.3), we have

$$
(-1)^{n-1} \tilde{p}_{\mu_{0}}(-1)>0
$$

Let us define a set of determinants $\widetilde{\Delta}_{j}^{ \pm}(\mu)$ of $\tilde{p}_{\mu}(\lambda), j=n-2, n-3, \ldots, 1,0$, similar to (3.2). We then substitute (3.6) into $\Delta_{j}^{ \pm}\left(\mu_{0}\right), j=n-2, n-4, \ldots, 1$ or 2 , and make the elementary row operations for each row of $\Delta_{j}^{ \pm}\left(\mu_{0}\right)$ as follows: starting from the last row of $\Delta_{j}^{ \pm}\left(\mu_{0}\right)$, multiply the $m$ th row by -1 , and add it to the $(m-1)$ th row (if any), to obtain

$$
\Delta_{j}^{ \pm}\left(\mu_{0}\right)=\widetilde{\Delta}_{j}^{ \pm}\left(\mu_{0}\right), \quad j=n-2, n-4, \ldots, 1, \text { or } 2 .
$$

Notice that Lemma 3.1 is proposed for an $n$-dimension map in a general sense, and it is applicable for the $(n-1)$-order characteristic polynomial $\tilde{p}_{\mu}(\lambda)$ with minor modification. 
According to (3.8), the equalities and inequalities in the condition (T1), except for $p_{\mu_{0}}(-1)=$ 0 , implies the fact that the polynomial $\widetilde{p}_{\mu_{0}}(\lambda)=0$ satisfies with the condition (H1). By considering the additional condition equation (3.7), we can readily infer the eigenvalue assignment of the polynomial $\tilde{p}_{\mu_{0}}(\lambda)=0$ that at $\mu=\mu_{0}$ a pair of complex conjugate eigenvalue lie on the unit circle and the others lie in the unit disk. The condition (C1) thus is satisfied under the condition (T1). Inversely, based on (3.5)-(3.8), one can conclude that the condition (T1) holds if the Jacobian matrix $D_{x_{k}} f_{\mu}\left(x_{0}\right)$ has the eigenvalue assignment stated in (C1).

The transversality condition $(\mathrm{C} 2)$ means that the -1 eigenvalue and the complex conjugate eigenvalues $\lambda_{1}\left(\mu_{0}\right)$ and $\bar{\lambda}_{1}\left(\mu_{0}\right)$ lying on the unit circle will cross the unit circle at nonzero rate if $\mu$ varies near $\mu_{0}$. Note that with $\lambda_{3}\left(\mu_{0}\right)=-1$, we have $\partial\left|\lambda_{3}(\mu)\right| /\left.\partial \mu_{j}\right|_{\mu=\mu_{0}}=$ $-\partial \lambda_{3}(\mu) /\left.\partial \mu_{j}\right|_{\mu=\mu_{0}}, j=1,2$. Directly differentiating the two sides of the characteristic polynomial $p_{\mu}(\lambda)=0$ with respect to $\mu_{j}$ at $\mu=\mu_{0}$ and substituting $\lambda_{3}\left(\mu_{0}\right)$ for $\lambda$, we find that $\partial\left|\lambda_{3}(\mu)\right| /\left.\partial \mu_{j}\right|_{\mu=\mu_{0}} \neq 0$ leads to (3.4) and vice versa. In addition, (3.5) implies that the sole pair of complex conjugate eigenvalues $\lambda_{1}\left(\mu_{0}\right)$ and $\bar{\lambda}_{1}\left(\mu_{0}\right)$ lying on the unit circle of $p_{\mu_{0}}(\lambda)=0$ are just the ones of $\tilde{p}_{\mu_{0}}(\lambda)=0$. As in the case of the derivation of equalities (3.8), we can obtain $\Delta_{n-2}^{-}(\mu)=\widetilde{\Delta}_{n-2}^{-}(\mu)$ such that $\partial \Delta_{n-2}^{-}(\mu) /\left.\partial \mu_{j}\right|_{\mu=\mu_{0}} \neq 0$ means $\partial \widetilde{\Delta}_{n-2}^{-}(\mu) /\left.\partial \mu_{j}\right|_{\mu=\mu_{0}} \neq 0$. It follows from Lemma 3.1 that $(\mathrm{H} 2)$ is equivalent to (D2). Note that this conclusion holds for the pair of complex conjugate eigenvalues of $\tilde{p}_{\mu_{0}}(\lambda)=0$ lying on the unit circle as well. Therefore, (T2) is equivalent to $(\mathrm{C} 2)$.

Finally, according to Lemma 3.1, the nonresonance condition of $\tilde{p}_{\mu_{0}}(\lambda)=0$ with respect to the pair of complex conjugate eigenvalues lying on the unit circle should be $\cos (2 \pi / m) \neq \psi$ where $m=3,4,5, \ldots$ and

$$
\psi=1-\frac{0.5 \tilde{p}_{\mu_{0}}(1) \widetilde{\Delta}_{n-4}^{-}\left(\mu_{0}\right)}{\tilde{\Delta}_{n-3}^{+}\left(\mu_{0}\right)} .
$$

Equations (3.5) and (3.8) give $p_{\mu_{0}}(1)=2 \widetilde{p}_{\mu_{0}}(1)$ and $\Delta_{n-4}^{-}\left(\mu_{0}\right)=\widetilde{\Delta}_{n-4}^{-}\left(\mu_{0}\right)$, respectively. By using the same procedures in the derivation of the equalities in (3.8), we can obtain $\Delta_{n-3}^{-}\left(\mu_{0}\right)=$ $\tilde{\Delta}_{n-3}^{-}\left(\mu_{0}\right)$. Thus, (3.9) can be rewritten as,

$$
\psi=1-\frac{p_{\mu_{0}}(1) \Delta_{n-4}^{-}\left(\mu_{0}\right)}{4 \Delta_{n-3}^{+}\left(\mu_{0}\right)} .
$$

It follows from (3.10) that the condition (T3) is equivalent to (C3).

It should be mentioned that different from the conditions $(\mathrm{C} 1)-(\mathrm{C} 3)$, the conditions (T1)-(T3) are formulated as a set of simple equalities or inequalities, explore the effects of the two parameters on the bifurcation and are independent of the computations of all eigenvalues. For example, for a three-dimension map, we have the following Corollary 3.3.

Corollary 3.3. For map (2.1) with $n=3$ to appear a flip-Neimark-Sacker bifurcation at $\mu_{0}$, it is necessary and sufficient that the following conditions (E1)-(E2) hold:

(E1) eigenvalue assignment: $a_{3}=1, a_{1}=a_{2},-1<a_{1}<3$; 
(E2) transversality condition:

$$
\begin{gathered}
a_{3 j}^{\prime} \neq 0, \quad j=1,2, \\
\frac{a_{1 j}^{\prime}-a_{2 j}^{\prime}+a_{3 j}^{\prime}}{a_{0}-2 a_{1}+a_{2}} \neq 0, \quad j=1,2,
\end{gathered}
$$

where $a_{i j}^{\prime}$ stands for the derivative of $a_{i}(\mu)$ with respect to $\mu_{j}$ at $\mu=\mu_{0}$;

(E3) nonresonance condition: $\cos (2 \pi / m) \neq \psi$ where $m=3,4,5, \ldots$, and $\psi=\left(3-a_{1}-a_{2}-a_{3}\right) / 4$.

Here, we briefly show the procedures to obtain the eigenvalue assignment (E1) in term of the conditions in (T1). Notice that the condition $\Delta_{n-2}^{-}\left(\mu_{0}\right)=0$ gives $a_{3}=1$. The condition $p_{\mu_{0}}(-1)=0$ gives $-1+a_{1}-a_{2}+a_{3}=0$, which implies $a_{1}=a_{2}$. It is from $p_{\mu_{0}}(1)>0$ that $1+a_{1}+a_{2}+a_{3}>0$. Then, we have $-1<a_{1}$ by using $a_{3}=1$ and $a_{1}=a_{2}$. The condition $(-1)^{n-1} \sum_{k=1}^{n}\left((-1)^{n-k} \sum_{i=1}^{k}\left((-1)^{k-i} a_{i-1}\right)\right)>0$ gives $3-2 a_{1}+a_{2}>0$, which implies $a_{1}<3$ on the basis of $a_{1}=a_{2}$. The condition $\Delta_{n-2}^{+}\left(\mu_{0}\right)>0$ representing $a_{3}>-1$ is superfluous due to $a_{3}=1$.

It is clear from Corollary 3.3 that the proposed criterion is formulated as a set of simple equalities or inequalities. The inequalities may directly exclude some parameter region in the parameter plane and suggest no need for computing all eigenvalues point by point in the parameter plane. This implies that the significant computational simplification for anticontrolling or detecting the codimension-two bifurcation parameter point. In addition, the potential of the criterion to reveal the effect of multiparameter on the bifurcation is illustrated by numerical examples in the next section.

\section{Numerical Examples}

The proposed critical criterion in Section 3 is applicable for any high-dimensional map in a general sense, such as the Poincaré map of impact vibrators [21], the three-order RodriguezVazquez map [25] and the generalized Hénon map [26]. The feasibility of the critical criterion in theoretical analysis and practical applications is illustrated by the following two examples.

Example 4.1. By incorporating a washout-filter controller into the four-dimension generalized Hénon map [19], a five-dimension control system takes the following form:

$$
\begin{aligned}
& x_{1}(k+1)=0.75-x_{4}(k)^{2}-0.1 x_{3}(k)+\mu_{1}\left(x_{2}(k)-1.5 w(k)\right), \\
& x_{2}(k+1)=x_{1}(k), \\
& x_{3}(k+1)=x_{2}(k), \\
& x_{4}(k+1)=x_{3}(k)+\mu_{2}\left(x_{2}(k)-1.5 w(k)\right), \\
& w(k+1)=x_{2}(k)-0.5 w(k),
\end{aligned}
$$

where the gains $\mu_{1} \in[-1.8,1]$ and $\mu_{2} \in[-0.5,3]$ to be determined stand for the bifurcation parameters in order of anticontrol of this bifurcation. Map (4.1) has a fixed point 


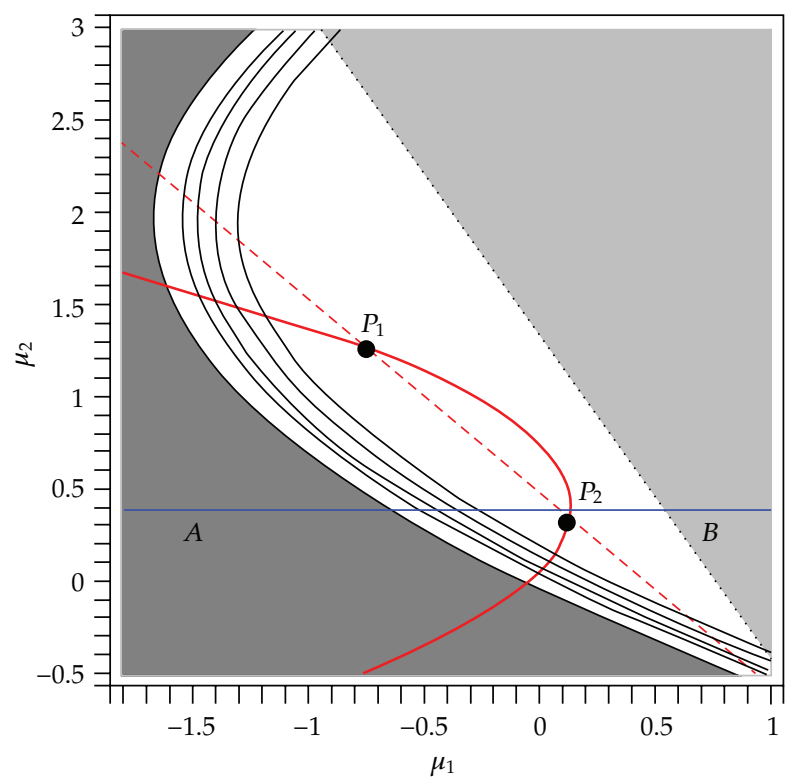

Figure 1: The illustrated conditions (T1)-(T3) of matrix (4.2) in Example 4.1. The blank region denotes the parameter domain in which all inequalities in the condition (T1) hold whereas in the gray region at least one inequality fails. The red heavy solid line and the red break line stand for $\Delta_{3}^{-}\left(\mu_{0}\right)=0$ and $p_{\mu_{0}}(-1)=0$, respectively. Their intersecting points are marked with $P_{1}$ and $P_{2}$. The other lines crossing the blank region consist of the parameter points where the transversality condition (T2) or the nonresonance condition (T3) fails.

$x_{0}=\left(x_{10}, x_{10}, x_{10}, x_{10}, w_{0}\right)^{T}$ with $w_{0}=x_{10} / 1.5$ and $x_{10}=0.475914$. The following matrix $D_{x_{k}} f_{\mu}\left(x_{0}\right)$ denotes the Jacobian matrix of map (4.1) at the fixed point $x_{0}$ :

$$
D_{x_{k}} f_{\mu}\left(x_{0}\right)=\left[\begin{array}{ccccc}
0 & \mu_{1} & -1 & -0.95182845 & -1.5 \mu_{1} \\
1 & 0 & 0 & 0 & 0 \\
0 & 1 & 0 & 0 & 0 \\
0 & \mu_{2} & 1 & 0 & -1.5 \mu_{2} \\
0 & 1 & 0 & 0 & -0.5
\end{array}\right]
$$

The conditions (T1)-(T3) are described in Figure 1. The blank region denotes the parameter domain in which all inequalities in the condition (T1) hold whereas in the gray region at least one inequality fails. Notice that we need not compute all eigenvalues point by point in the two parameter domain (the gray region in Figure 1), which is picked off by two inequalities in (T1)-(T3). It should be mentioned that, not only for the bifurcation parameters, but also for other system parameters having this characteristic in parameter analysis. The red heavy solid line and the red break line stand for $\Delta_{3}^{-}\left(\mu_{0}\right)=0$ and $p_{\mu_{0}}(-1)=0$, respectively. Their intersecting points are marked with $P_{1}$ and $P_{2}$. The other black lines crossing the blank region consist of the parameter points where the transversality condition (T2) or the nonresonance condition (T3) fails. Based on Theorem 3.2, it is clear that both point $P_{1}(-0.725229,1.248199)$ and point $P_{2}(0.136024,0.343479)$ are the bifurcation parameter points of the flip-Neimark-Sacker bifurcations for map (4.1). It should be noted that the blue straight line $A B$, which stands for the parameter points with $\partial \Delta_{3}^{-}(\mu) /\left.\partial \mu_{1}\right|_{\mu=\mu_{0}}=0$, is very close to 


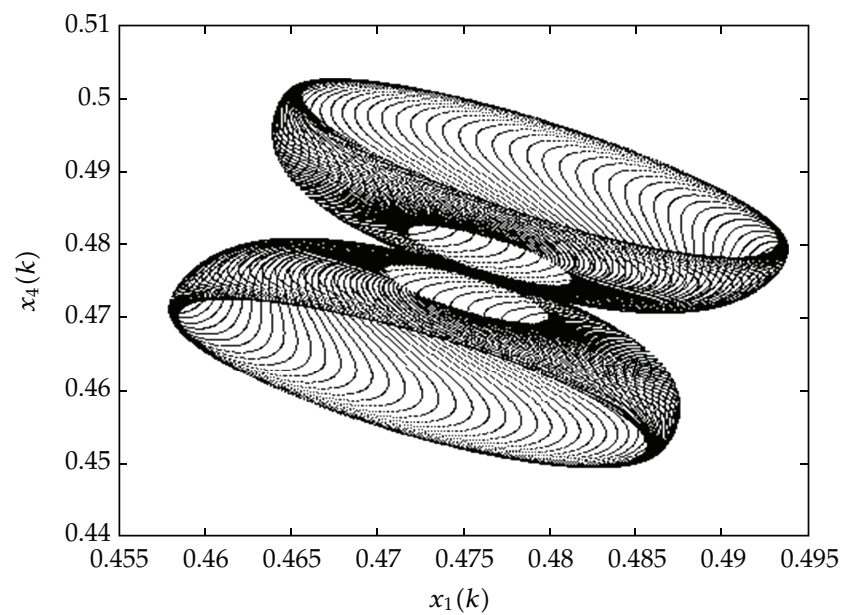

Figure 2: The typical bifurcation solution resulting from the flip-Neimark-Sacker bifurcation of map (4.1).

the bifurcation parameter point $P_{2}$ such that the change of the parameter $\mu_{1}$ is not sensitive for the complex conjugate eigenvalues lying on the unit circle at $P_{2}$ to cross the unit circle. Therefore, the ideal bifurcation parameter point anticontrol of this bifurcation is accurately located at the point $P_{1}(-0.725229,1.248199)$.

Furthermore, to demonstrate the bifurcation parameter point $P_{1}$, the simulation results of map (3.2) are used to show the bifurcation solutions. We take the bifurcation parameters $\left(\mu_{1}, \mu_{2}\right)=P_{1}+(-0.0001,0.0001)$ in the small neighborhood of the bifurcation parameter point $P_{1}$, and the very small perturbation of the fixed point $x_{0}$ as the initial iteration point. The total iteration number is 50000. One of the simulation results for map (4.1) is presented in Figure 2, in which a pair of stable invariant circles represents one of the most interesting bifurcation solutions of flip-Neimark-Sacker bifurcations.

Example 4.2. By applying the forward Euler scheme with step-size of the form $h=1 / 5$ to discretize the sunflower model with delay [27], we can obtain a twelve-dimension nonlinear map. Its Jacobian matrix $D_{x_{k}} f_{\mu}\left(x_{0}\right)$ takes the following form:

$$
D_{x_{k}} f_{\mu}\left(x_{0}\right)=\left(\begin{array}{cccccccccccc}
1 & r h & 0 & 0 & 0 & 0 & 0 & 0 & 0 & 0 & 0 & 0 \\
0 & 1-\mu_{1} h & 0 & 0 & 0 & 0 & 0 & 0 & 0 & -\mu_{2} h & 0 & 0 \\
1 & 0 & 0 & 0 & 0 & 0 & 0 & 0 & 0 & 0 & 0 & 0 \\
0 & 1 & 0 & 0 & 0 & 0 & 0 & 0 & 0 & 0 & 0 & 0 \\
0 & 0 & 1 & 0 & 0 & 0 & 0 & 0 & 0 & 0 & 0 & 0 \\
0 & 0 & 0 & 1 & 0 & 0 & 0 & 0 & 0 & 0 & 0 & 0 \\
0 & 0 & 0 & 0 & 1 & 0 & 0 & 0 & 0 & 0 & 0 & 0 \\
0 & 0 & 0 & 0 & 0 & 1 & 0 & 0 & 0 & 0 & 0 & 0 \\
0 & 0 & 0 & 0 & 0 & 0 & 1 & 0 & 0 & 0 & 0 & 0 \\
0 & 0 & 0 & 0 & 0 & 0 & 0 & 1 & 0 & 0 & 0 & 0 \\
0 & 0 & 0 & 0 & 0 & 0 & 0 & 0 & 1 & 0 & 0 & 0 \\
0 & 0 & 0 & 0 & 0 & 0 & 0 & 0 & 0 & 1 & 0 & 0
\end{array}\right)
$$




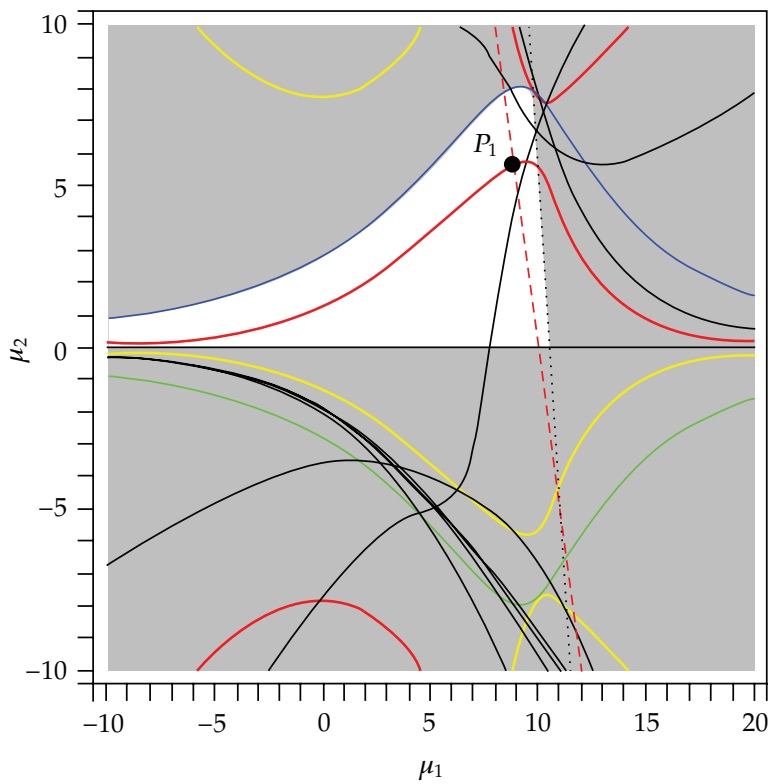

Figure 3: The illustrated conditions (T1)-(T3) of matrix (4.3) in Example 4.2.

where the delay $r=2$, and the bifurcation parameter vector $\mu$ consists of the two unknown parameters $\mu_{1}$ and $\mu_{2}$, that is, $\mu=\left(\mu_{1}, \mu_{2}\right)^{T}$. For the above high-dimensional system with two parameters to be determined, acquiring the explicit forms of all eigenvalues is extremely difficult, or even infeasible. However, with the aid of Theorem 3.2 and the software Maple or Matlab, we can easily graph the conditions (T1)-(T3) as shown in Figure 3. Similar to Figure 1, the blank region denotes the parameter domain in which all inequalities in the condition (T1) hold whereas in the gray region at least one inequality fails. The red heavy solid line and the red break line with an intersecting point $P_{1}$ stand for $\Delta_{10}^{-}\left(\mu_{0}\right)=0$ and $p_{\mu_{0}}(-1)=0$, respectively. The other black lines crossing the blank region consist of the parameter points where the transversality condition (T2) or the nonresonance condition (T3) fails. In Figure 3, it is evident that the point $P_{1}$ is the bifurcation parameter point of the flip-Neimark-Sacker bifurcation.

It should be mentioned that if the classical critical criterion stated in Definition 2.1 is used to seek the bifurcation point $P_{1}$, we have to numerically compute all eigenvalues of matrix (4.3) point by point scanning the parameter plane such that the work seems like seeking a needle in the meadow. However, as shown in Figure 3, only the condition $p_{\mu_{0}}(1)>0$ in Theorem 3.2 may exclude half of the parameter region in the process of obtaining the bifurcation point $P_{1}$. Obviously, with the significant computational simplification, the proposed bifurcation critical criterion in Theorem 3.2 is more efficient and convenient in detecting the existence of the bifurcation.

Finally, let us show another advantage of Theorem 3.2: its potential to reveal the effect of multiparameter on the bifurcation. For example, we locally zoom in a region near the point $P_{1}$ in Figure 3 and show it in Figure 4. One can easily check the fact that $p_{\mu_{0}}(-1)<0$ and $\Delta_{10}^{-}\left(\mu_{0}\right)<0$ hold in region I, $p_{\mu_{0}}(-1)<0$ and $\Delta_{10}^{-}\left(\mu_{0}\right)>0$ in region II, $p_{\mu_{0}}(-1)>0$ and $\Delta_{10}^{-}\left(\mu_{0}\right)>0$ in region III; and $p_{\mu_{0}}(-1)>0$ and $\Delta_{10}^{-}\left(\mu_{0}\right)<0$ in region IV. This implies that at a sufficient small parameter neighborhood of the point $P_{1}$, a couple of invariant circles as one 


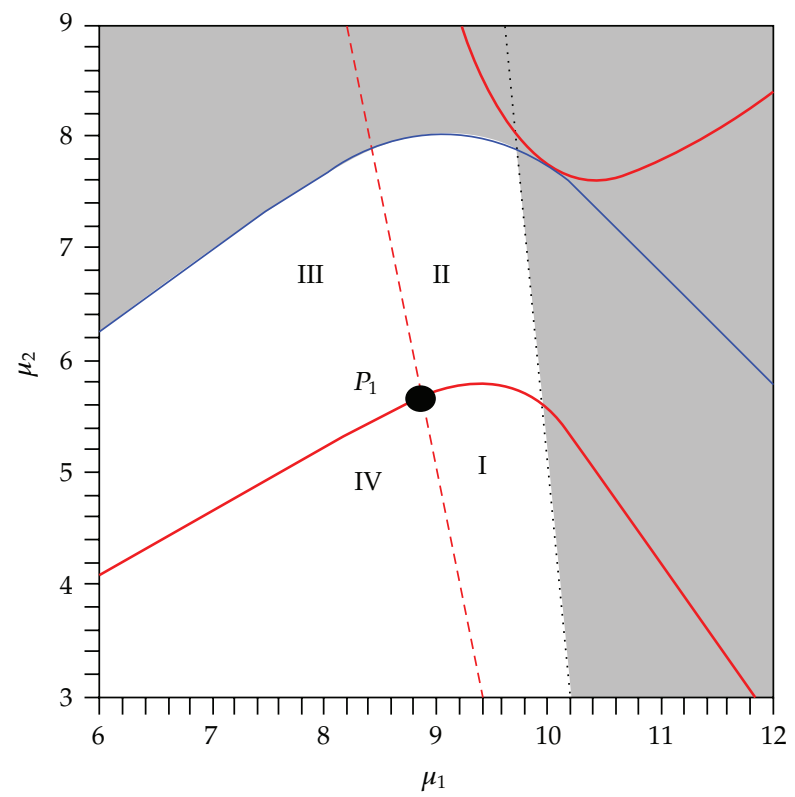

Figure 4: The enlargement of a region near the point $P_{1}$ in Figure 3. The red heavy solid line and the red break line partition off the blank region to four subregions, labeled as I, II, III and IV, respectively.

invariant set may emerge in region I, fixed points of order 2 exist in region I, single-limit circle appears in region III, and the original fixed point $x_{0}$ is stable (no bifurcation occurs) in region II. In other words, Figure 4 gives the direction of parameter unfolding at the sufficient small neighborhood of the point $P_{1}$.

\section{Conclusions and Remarks}

A new criterion without using eigenvalues is proposed for flip-Neimark-Sacker bifurcation of a general discrete time system (or map). With the application of the criterion, the effects of parameters on the bifurcation in two-parameterized family of $n$-dimensional discrete systems may be explicitly formulated. The proposed critical criterion is more convenient and efficient for analyzing or anticontrolling this complicated codimension-two bifurcation than the classical critical criterion, especially for high-dimensional maps.

\section{References}

[1] G. Iooss, Bifurcation of Maps and Applications, vol. 36 of North-Holland Mathematics Studies, NorthHolland, Amsterdam, The Netherlands, 1979.

[2] Y. A. Kuznetsov, Elements of Applied Bifurcation Theory, vol. 112 of Applied Mathematical Sciences, Springer, New York, NY, USA, 2nd edition, 1998.

[3] J. Guckenheimer and P. Holmes, Nonlinear Oscillations, Dynamical Systems, and Bifurcations of Vector Fields, vol. 42 of Applied Mathematical Sciences, Springer, New York, NY, USA, 1990.

[4] G. Chen, D. J. Hill, and X. H. Yu, Bifurcation Control: Theory and Applications, vol. 293 of Lecture Notes in Control and Information Sciences, Springer, Berlin, Germany, 2003.

[5] G. Chen, Controlling Chaos and Bifurcations in Engineering Systems, CRC Press, Boca Raton, Fla, USA, 1999. 
[6] D. S. Chen, H. O. Wang, and G. Chen, "Anti-control of Hopf bifurcations," IEEE Transactions on Circuits and Systems. I, vol. 48, no. 6, pp. 661-672, 2001.

[7] G. Chen, J. Lu, B. Nicholas, and S. M. Ranganathan, "Bifurcation dynamics in discrete-time delayedfeedback control systems," International Journal of Bifurcation and Chaos in Applied Sciences and Engineering, vol. 9, no. 1, pp. 287-293, 1999.

[8] A. Dhooge, W. Govaerts, and Yu. A. Kuznetsov, "MATCONT: a MATLAB package for numerical bifurcation analysis of ODEs," ACM Transactions on Mathematical Software, vol. 29, no. 2, pp. 141-164, 2003.

[9] G. Wen, "Criterion to identify Hopf bifurcations in maps of arbitrary dimension," Physical Review E, vol. 72, no. 2, Article ID 026201, 4 pages, 2005.

[10] G. Wen and D. Xu, "Control algorithm for creation of Hopf bifurcations in continuous-time systems of arbitrary dimension," Physics Letters, Section A, vol. 337, no. 1-2, pp. 93-100, 2005.

[11] J. P. Lasalle, The Stability and Control of Discrete Processes, vol. 62 of Applied Mathematical Sciences, Springer, New York, NY, USA, 1986.

[12] B. M. Brown, "On the distribution of the zeros of a polynomial," The Quarterly Journal of Mathematics, vol. 16, pp. 241-256, 1965.

[13] B. C. Kuo, Analysis and Synthesis of Sampled-Data Control Systems, Prentice-Hall, Englewood Cliffs, NJ, USA, 1963.

[14] G. Wen, D. Xu, and X. Han, "On creation of Hopf bifurcations in discrete-time nonlinear systems," Chaos, vol. 12, no. 2, pp. 350-355, 2002.

[15] G. Wen and D. Xu, "Designing Hopf bifurcations into nonlinear discrete-time systems via feedback control," International Journal of Bifurcation and Chaos, vol. 14, no. 7, pp. 2283-2293, 2004.

[16] G. Wen, D. Xu, and J.-H. Xie, "Controlling Hopf bifurcations of discrete-time systems in resonance," Chaos, Solitons and Fractals, vol. 23, no. 5, pp. 1865-1877, 2005.

[17] G. Wen, S. Chen, and Q. Jin, "A new criterion of period-doubling bifurcation in maps and its application to an inertial impact shaker," Journal of Sound and Vibration, vol. 311, no. 1-2, pp. 212-223, 2008.

[18] G. Wen, D. Xu, and J. Xie, "Control of degenerate Hopf bifurcations in three-dimensional maps," Chaos, vol. 13, no. 2, pp. 486-494, 2003.

[19] G.-L. Wen and D. Xu, "Feedback control of Hopf-Hopf interaction bifurcation with development of torus solutions in high-dimensional maps," Physics Letters. A, vol. 321, no. 1, pp. 24-33, 2004.

[20] G. Wen, H. Xu, and J.-H Xie, "Controlling Hopf-Hopf interaction bifurcations of a two-degree-offreedom self-excited system with dry friction," Nonlinear Dynamics, vol. 64, no. 1-2, pp. 49-57, 2011.

[21] G. Wen, H. Xu, and Z. Chen, "Anti-controlling quasi-periodic impact motion of an inertial impact shaker system," Journal of Sound and Vibration, vol. 329, no. 19, pp. 4040-4047, 2010.

[22] G. Iooss and J. E. Los, "Quasi-genericity of bifurcations to high-dimensional invariant tori for maps," Communications in Mathematical Physics, vol. 119, no. 3, pp. 453-500, 1988.

[23] W.-C. Ding, J.-H. Xie, and Q. G. Sun, "Interaction of Hopf and period doubling bifurcations of a vibroimpact system," Journal of Sound and Vibration, vol. 275, no. 1-2, pp. 27-45, 2004.

[24] G. Wen and D. Xu, "Implicit criteria of eigenvalue assignment and transversality for bifurcation control in four-dimensional maps," International Journal of Bifurcation and Chaos, vol. 14, no. 10, pp. 3489-3503, 2004.

[25] A. Rodriguez-Vazquez, J. L. Huertas, A. Rueda, B. Perez-Verdu, and L. O. Chua, "Chaos from switched-capacitor circuits: discrete maps," Proceedings of the IEEE, vol. 75, no. 8, pp. 1090-1106, 1987.

[26] G. Grassi and D. A. Miller, "Theory and experimental realization of observer-based discrete-time hyperchaos synchronization," IEEE Transactions on Circuits and Systems I, vol. 49, no. 3, pp. 373-378, 2002.

[27] C. Zhang, M. Liu, and B. Zheng, "Hopf bifurcation in numerical approximation of a class delay differential equations," Applied Mathematics and Computation, vol. 146, no. 2-3, pp. 335-349, 2003. 


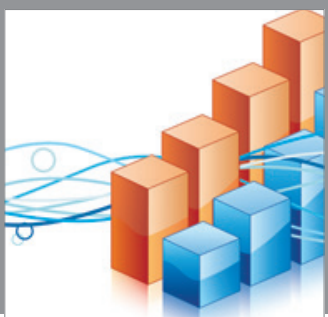

Advances in

Operations Research

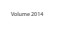

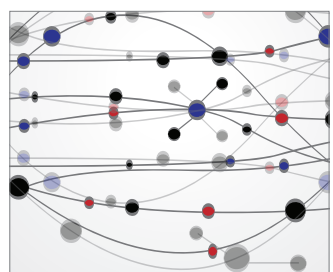

\section{The Scientific} World Journal
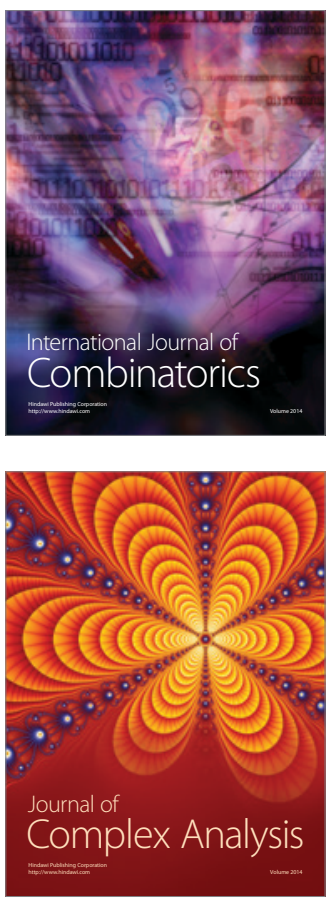

International Journal of

Mathematics and

Mathematical

Sciences
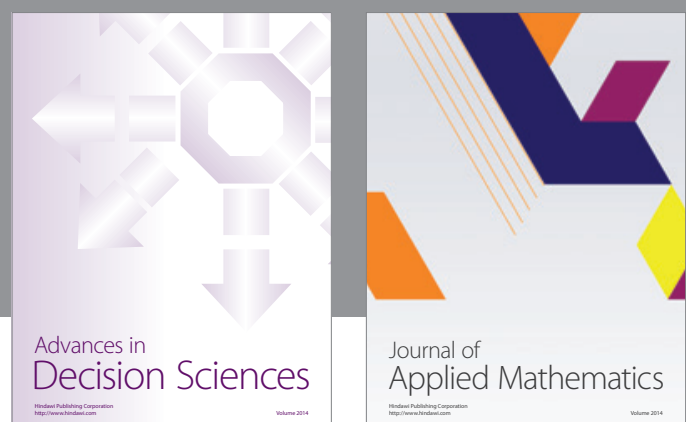

Journal of

Applied Mathematics
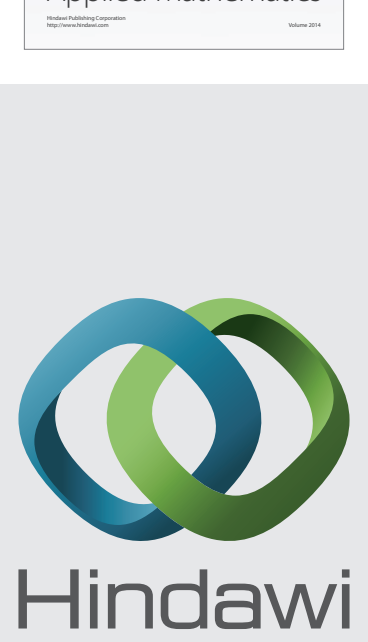

Submit your manuscripts at http://www.hindawi.com
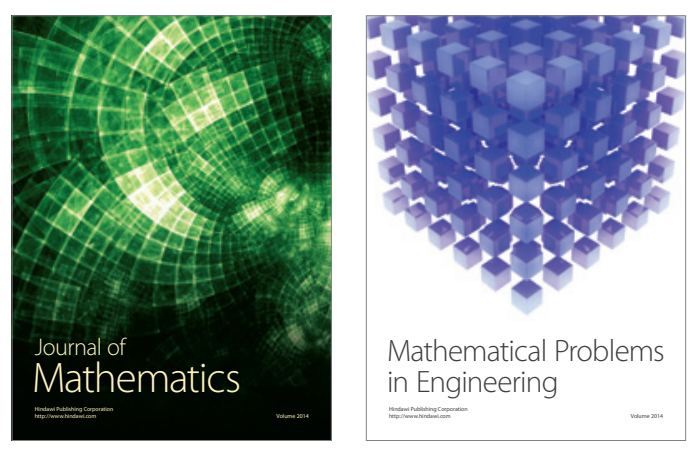

Mathematical Problems in Engineering
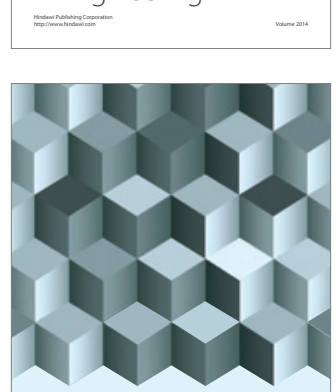

Journal of

Function Spaces
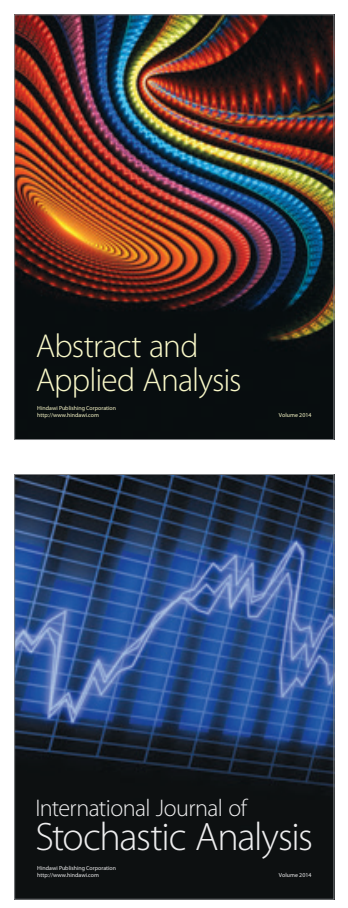

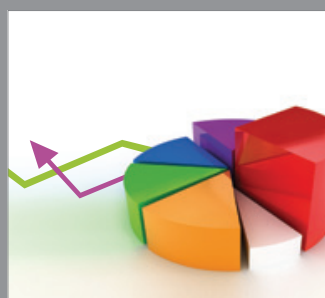

ournal of

Probability and Statistics

Promensencen
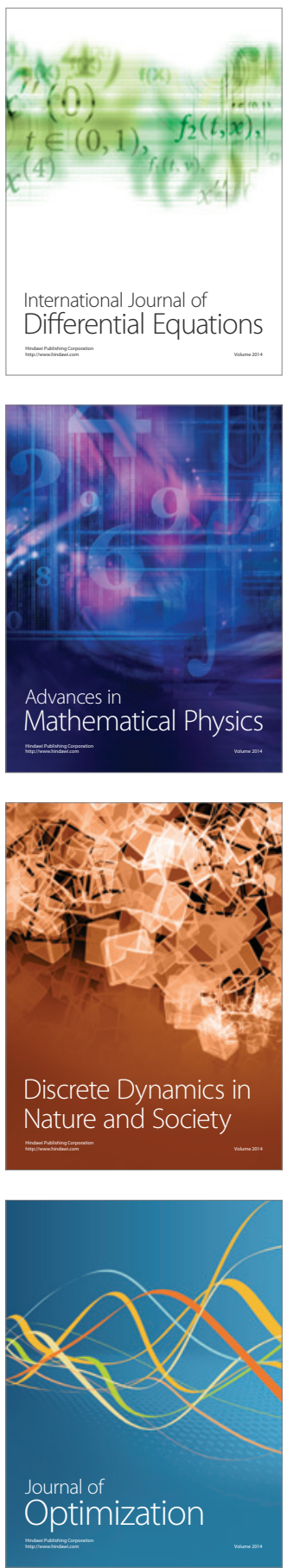